Acta Crystallographica Section E

Structure Reports

Online

ISSN 1600-5368

Krishnan Srinivasan, a Subbaiah Govindarajan $^{\mathrm{a}}$ and William T. A. Harrison $^{\mathrm{b}}$ *

a Department of Chemistry, Bharathiar University, Coimbatore 641 046, India, and ${ }^{\mathbf{b}}$ Department of Chemistry, University of Aberdeen, Meston Walk, Aberdeen AB24 3UE, Scotland

Correspondence e-mail: w.harrison@abdn.ac.uk

\section{Key indicators}

Single-crystal X-ray study

$T=120 \mathrm{~K}$

Mean $\sigma(\mathrm{N}-\mathrm{N})=0.004 \AA$

$R$ factor $=0.032$

$w R$ factor $=0.076$

Data-to-parameter ratio $=11.2$

For details of how these key indicators were automatically derived from the article, see http://journals.iucr.org/e.

\title{
Iron(II) hydrazinium sulfate
}

The title compound, poly[[dihydraziniumiron(II)]-di- $\mu$ sulfato- $\left.\kappa^{4} O: O^{\prime}\right],\left[\mathrm{Fe}\left(\mathrm{SO}_{4}\right)_{2}\left(\mathrm{~N}_{2} \mathrm{H}_{5}\right)_{2}\right]_{n}$, contains fairly regular trans $-\mathrm{FeN}_{2} \mathrm{O}_{4}$ octahedra. The $\mathrm{Fe}$ atoms (site symmetry $\overline{1}$ ) are bridged by pairs of sulfate groups into infinite [100] chains, which are cross-linked by a network of $\mathrm{N}-\mathrm{H} \cdots \mathrm{O}$ hydrogen bonds. $\mathrm{Fe}\left(\mathrm{N}_{2} \mathrm{H}_{5}\right)_{2}\left(\mathrm{SO}_{4}\right)_{2}$ is isostructural with its zinc, chromium(II) and cadmium-containing analogues.

\section{Comment}

The divalent-metal-hydrazinium sulfates of general formula $M\left(\mathrm{~N}_{2} \mathrm{H}_{5}\right)_{2}\left(\mathrm{SO}_{4}\right)_{2}$, where $M=\mathrm{Cr}, \mathrm{Mn}, \mathrm{Fe}, \mathrm{Co}, \mathrm{Ni}, \mathrm{Cu}, \mathrm{Zn}$ and $\mathrm{Cd}$ can be readily prepared by reacting a salt of the respective metal with hydrazinium sulfate in dilute sulfuric acid (Hand \& Prout, 1966), although this method usually results in a microcrystalline product. Recently, we described the singlecrystal structure of $\mathrm{Cd}\left(\mathrm{N}_{2} \mathrm{H}_{5}\right)_{2}\left(\mathrm{SO}_{4}\right)_{2}$ (Srinivasan et al., 2006) and we now report the isostructural title compound, (I), $\mathrm{Fe}\left(\mathrm{N}_{2} \mathrm{H}_{5}\right)_{2}\left(\mathrm{SO}_{4}\right)_{2}$. The compounds $\mathrm{Zn}\left(\mathrm{N}_{2} \mathrm{H}_{5}\right)_{2}\left(\mathrm{SO}_{4}\right)_{2}$ (Prout \& Powell, 1961) and $\mathrm{Cr}\left(\mathrm{N}_{2} \mathrm{H}_{5}\right)_{2}\left(\mathrm{SO}_{4}\right)_{2}$ (Parkins et al., 2001) also share the same stucture.

Compound (I) contains trans- $\mathrm{FeN}_{2} \mathrm{O}_{4}$ octahedra (Fig. 1), in which the $\mathrm{N}$ atom is part of a hydrazinium $\left(\mathrm{N}_{2} \mathrm{H}_{5}{ }^{+}\right)$cation. The Fe atoms (site symmetry $\overline{1}$ ) are connected by pairs of sulfate groups into infinite chains that propagate in [100]. The intrachain $\mathrm{Fe} \cdots \mathrm{Fe}$ separation in (I) is equal to the $a$ unit-cell dimension, i.e. 5.3306 (3) $\AA$. The two distinct $\mathrm{Fe}-\mathrm{O}$ bond lengths in (I) are similar (Table 1) and do not show the gross differences seen in the chromium and zinc analogues.

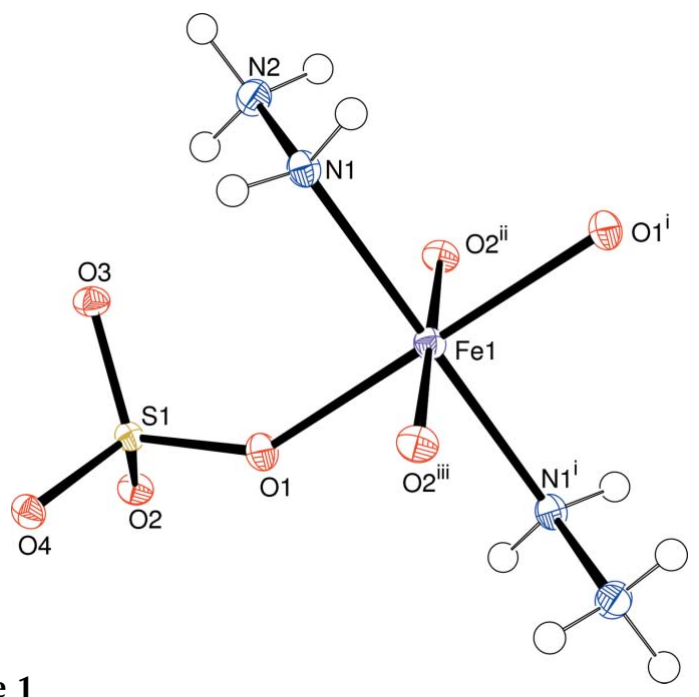

\section{Figure 1}

The asymmetric unit of (I) expanded to show the iron coordination $(50 \%$ displacement ellipsoids; arbitrary spheres for the $\mathrm{H}$ atoms). Symmetry codes: (i) $-x,-y,-z$; (ii) $x-1, y, z$; (iii) $1-x,-y,-z$.
Received 22 December 2006 Accepted 29 December 2006 
The iron-sulfate chains in (I) are cross-linked by $\mathrm{N}-\mathrm{H} \cdots \mathrm{O}$ hydrogen bonds (Table 2), resulting in the same hydrogenbonding network seen in the other analogues noted above. A well defined trifurcated $\mathrm{N} 2-\mathrm{H} 3 C \cdots(\mathrm{O}, \mathrm{O}, \mathrm{O})$ interaction occurs (mean bond angle about $\mathrm{H} 3 C=107.3^{\circ}$ ).

\section{Experimental}

The reaction of hydrazine monohydrate $\left(\mathrm{N}_{2} \mathrm{H}_{4} \cdot \mathrm{H}_{2} \mathrm{O} ; 0.50 \mathrm{~g}, 10 \mathrm{mmol}\right)$ and ethyl bromoacetate $(1.671 \mathrm{~g}, 10 \mathrm{mmol})$ in $5 \mathrm{ml}$ of dry ethanol resulted in the formation of a white solid containing hydrazinium bromide and ethyl hydrazinoacetate, as reported earlier (Srinivasan et al., 2006). This white solid $(0.236 \mathrm{~g})$ was dissolved in water $(30 \mathrm{ml})$ and mixed with an aqueous solution $(30 \mathrm{ml})$ of $\mathrm{FeSO}_{4} \cdot 7 \mathrm{H}_{2} \mathrm{O}(0.278 \mathrm{~g}$, $1 \mathrm{mmol}$ ) and a few drops of conc. $\mathrm{H}_{2} \mathrm{SO}_{4}$. The resulting clear solution, with a $\mathrm{pH}$ of 2 , was concentrated over a water bath to $20 \mathrm{ml}$ and kept for crystallization at room temperature. After three days, many block-shaped light-green crystals of (I) had formed. These were recovered by filtration, washed with cold water and dried in air.

\section{Crystal data}

$\begin{array}{ll}{\left[\mathrm{Fe}\left(\mathrm{SO}_{4}\right)_{2}\left(\mathrm{~N}_{2} \mathrm{H}_{5}\right)_{2}\right]} & V=219.41(2) \AA^{3} \\ M_{r}=314.08 & Z=1 \\ \text { Triclinic, } P \overline{1} & D_{x}=2.377 \mathrm{Mg} \mathrm{m}^{-3} \\ a=5.3306(3) \AA & \text { Mo } \mathrm{K} \alpha \text { radiation }^{-1} \\ b=5.8205(3) \AA & \mu=2.23 \mathrm{~mm}^{-1} \\ c=7.3835(4) \AA & T=120(2) \mathrm{K} \\ \alpha=92.034(3)^{\circ} & \text { Lath, pale green } \\ \beta=103.313(3)^{\circ} & 0.05 \times 0.02 \times 0.01 \mathrm{~mm} \\ \gamma=99.237(3)^{\circ} & \end{array}$

\section{Data collection}

Nonius KappaCCD diffractometer $\omega$ and $\varphi$ scans

Absorption correction: multi-scan (SADABS; Bruker, 2003)

$T_{\min }=0.897, T_{\max }=0.978$

\section{Refinement}

Refinement on $F^{2}$

$R\left[F^{2}>2 \sigma\left(F^{2}\right)\right]=0.032$

$w R\left(F^{2}\right)=0.076$

$S=1.12$

1004 reflections

90 parameters

All H-atom parameters refined

\section{Table 1}

Selected geometric parameters $\left(\AA{ }^{\circ}\right)$.

\begin{tabular}{lrlr}
\hline $\mathrm{Fe} 1-\mathrm{O} 1$ & $2.109(2)$ & $\mathrm{Fe} 1-\mathrm{N} 1$ & $2.184(2)$ \\
$\mathrm{Fe} 1-\mathrm{O} 2^{\mathrm{i}}$ & $2.147(2)$ & & \\
$\mathrm{S} 1-\mathrm{O} 1-\mathrm{Fe} 1$ & $142.94(13)$ & $\mathrm{S} 1-\mathrm{O} 2-\mathrm{Fe} 1^{\mathrm{ii}}$ & $128.85(12)$ \\
\hline
\end{tabular}

Symmetry codes: (i) $x-1, y, z$; (ii) $x+1, y, z$.

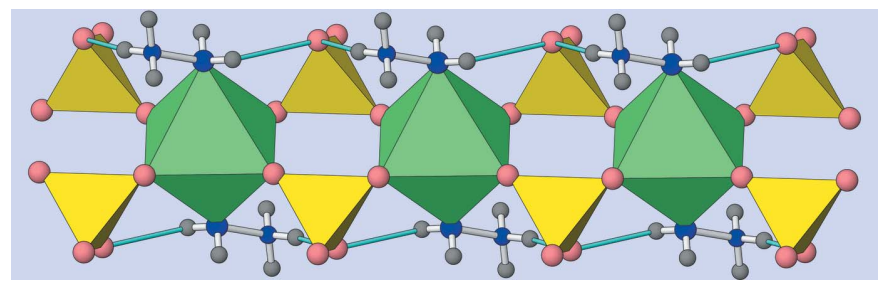

Figure 2

Polyhedral view of a fragment of the chain structure of (I). Colour key: $\mathrm{Fe}$ octahedra green, $\mathrm{S}$ tetrahedra yellow, $\mathrm{O}$ pink, $\mathrm{N}$ blue, $\mathrm{H}$ grey. The $\mathrm{H} \cdots \mathrm{O}$ portions of the hydrogen bonds are coloured light blue.

Table 2

Hydrogen-bond geometry $\left(\AA{ }^{\circ}\right)$.

\begin{tabular}{|c|c|c|c|c|}
\hline$D-\mathrm{H} \cdots A$ & $D-\mathrm{H}$ & $\mathrm{H} \cdots A$ & $D \cdots A$ & $D-\mathrm{H} \cdots A$ \\
\hline $\mathrm{N} 1-\mathrm{H} 1 A \cdots \mathrm{O} 3^{\mathrm{iii}}$ & $0.82(4)$ & $2.37(4)$ & $3.070(3)$ & $143(3)$ \\
\hline $\mathrm{N} 1-\mathrm{H} 1 B \cdots \mathrm{O} 4^{\mathrm{iv}}$ & $0.82(4)$ & 2.12 (4) & 2.867 (3) & $151(4)$ \\
\hline $\mathrm{N} 2-\mathrm{H} 2 A \cdots \mathrm{O} 4^{\mathrm{iii}}$ & $0.88(4)$ & $1.96(4)$ & 2.799 (3) & $160(4)$ \\
\hline $\mathrm{N} 2-\mathrm{H} 2 B \cdots \mathrm{O} 3$ & $0.80(4)$ & $2.02(4)$ & 2.769 (4) & $156(4)$ \\
\hline $\mathrm{N} 2-\mathrm{H} 2 C \cdots \mathrm{O} 2^{\mathrm{v}}$ & $0.82(4)$ & 2.51 (4) & $2.849(3)$ & $106(3)$ \\
\hline $\mathrm{N} 2-\mathrm{H} 2 C \cdots \mathrm{O} 2^{\mathrm{iv}}$ & $0.82(4)$ & $2.32(4)$ & 3.011 (4) & $141(4)$ \\
\hline $\mathrm{N} 2-\mathrm{H} 2 C \cdots \mathrm{O} 1^{\mathrm{vi}}$ & $0.82(4)$ & $2.45(4)$ & $3.073(3)$ & $133(4)$ \\
\hline
\end{tabular}

Symmetry codes: (iii) $-x+1,-y,-z+1$; (iv) $x-1, y-1, z$; (v) $-x+1,-y,-z$; (vi) $x, y-1, z$.

The $\mathrm{H}$ atoms were located in difference maps and their positions and $U_{\text {iso }}$ values were freely refined.

Data collection: Collect (Nonius, 1998); cell refinement: $H K L$ SCALEPACK (Otwinowski \& Minor 1997); data reduction: $H K L$ $D E N Z O$ and SCALEPACK (Otwinowski \& Minor 1997) \& SORTAV (Blessing 1995); program(s) used to solve structure: SHELXS97 (Sheldrick, 1997); program(s) used to refine structure: SHELXL97 (Sheldrick, 1997); molecular graphics: ORTEP-3 (Farrugia, 1997); software used to prepare material for publication: SHELXL97.

We thank the EPSRC National Crystallography Service (University of Southampton) for the data collection.

\section{References}

Blessing, R. H. (1995). Acta Cryst. A51, 33-38.

Bruker (2003). SADABS, Bruker AXS Inc., Madison, Wisconsin, USA.

Farrugia, L. J. (1997). J. Appl. Cryst. 30, 565.

Hand, D. W. \& Prout, C. K. (1966). J. Chem. Soc. A, pp. 168-171.

Nonius (1998). COLLECT. Nonius BV, Delft, The Netherlands.

Otwinowski, Z. \& Minor, W. (1997). Methods in Enzymology, Vol. 276, Macromolecular Crystallography, Part A, edited by C. W. Carter, Jr \& R. M. Sweet, pp. 307-326. New York: Academic Press.

Parkins, A. W., Prince, P. D., Smith, R. A. L. \& Steed, J. W. (2001). Acta Cryst. C57, 670-671.

Prout, C. K. \& Powell, H. M. (1961). J. Chem. Soc. pp. 4177-4182.

Sheldrick, G. M. (1997). SHELXS97 and SHELXL97. University of Göttingen, Germany.

Srinivasan, K., Govindarajan, S. \& Harrison, W. T. A. (2006). Acta Cryst. E62, i219-i221. 


\section{supporting information}

Acta Cryst. (2007). E63, i41-i42 [https://doi.org/10.1107/S1600536806056509]

Iron(II) hydrazinium sulfate

Krishnan Srinivasan, Subbaiah Govindarajan and William T. A. Harrison

(I)

\section{Crystal data}

$\mathrm{Fe}\left(\mathrm{N}_{2} \mathrm{H}_{5}\right)_{2}\left(\mathrm{SO}_{4}\right)_{2}$

$M_{r}=314.08$

Triclinic, $P \overline{1}$

Hall symbol: -P 1

$a=5.3306(3) \AA$

$b=5.8205(3) \AA$

$c=7.3835(4) \AA$

$\alpha=92.034(3)^{\circ}$

$\beta=103.313(3)^{\circ}$

$\gamma=99.237(3)^{\circ}$

$V=219.41(2) \AA^{3}$

Data collection

Nonius KappaCCD

diffractometer

Radiation source: fine-focus sealed tube

Graphite monochromator

$\omega$ and $\varphi$ scans

Absorption correction: multi-scan

(SADABS; Bruker, 2003)

$T_{\min }=0.897, T_{\max }=0.978$

Refinement

Refinement on $F^{2}$

Least-squares matrix: full

$R\left[F^{2}>2 \sigma\left(F^{2}\right)\right]=0.032$

$w R\left(F^{2}\right)=0.076$

$S=1.12$

1004 reflections

90 parameters

0 restraints

Primary atom site location: structure-invariant direct methods
$Z=1$

$F(000)=160$

$D_{\mathrm{x}}=2.377 \mathrm{Mg} \mathrm{m}^{-3}$

Mo $K \alpha$ radiation, $\lambda=0.71073 \AA$

Cell parameters from 984 reflections

$\theta=2.9-27.5^{\circ}$

$\mu=2.23 \mathrm{~mm}^{-1}$

$T=120 \mathrm{~K}$

Lath, pale green

$0.05 \times 0.02 \times 0.01 \mathrm{~mm}$

3957 measured reflections

1004 independent reflections

911 reflections with $I>2 \sigma(I)$

$R_{\text {int }}=0.043$

$\theta_{\max }=28.0^{\circ}, \theta_{\min }=3.6^{\circ}$

$h=-6 \rightarrow 6$

$k=-7 \rightarrow 7$

$l=-9 \rightarrow 9$

Secondary atom site location: difference Fourier map

Hydrogen site location: inferred from

neighbouring sites

All $\mathrm{H}$-atom parameters refined

$w=1 /\left[\sigma^{2}\left(F_{\mathrm{o}}^{2}\right)+(0.0127 P)^{2}+0.6538 P\right]$

where $P=\left(F_{\mathrm{o}}{ }^{2}+2 F_{\mathrm{c}}{ }^{2}\right) / 3$

$(\Delta / \sigma)_{\max }<0.001$

$\Delta \rho_{\max }=0.37 \mathrm{e} \AA^{-3}$

$\Delta \rho_{\min }=-0.58$ e $\AA^{-3}$

Special details

Geometry. All e.s.d.'s (except the e.s.d. in the dihedral angle between two 1.s. planes) are estimated using the full covariance matrix. The cell e.s.d.'s are taken into account individually in the estimation of e.s.d.'s in distances, angles and torsion angles; correlations between e.s.d.'s in cell parameters are only used when they are defined by crystal symmetry. An approximate (isotropic) treatment of cell e.s.d.'s is used for estimating e.s.d.'s involving 1.s. planes. 
Refinement. Refinement of $F^{2}$ against ALL reflections. The weighted $R$-factor $w R$ and goodness of fit $S$ are based on $F^{2}$, conventional $R$-factors $R$ are based on $F$, with $F$ set to zero for negative $F^{2}$. The threshold expression of $F^{2}>\sigma\left(F^{2}\right)$ is used only for calculating $R$-factors(gt) etc. and is not relevant to the choice of reflections for refinement. $R$-factors based on $F^{2}$ are statistically about twice as large as those based on $F$, and $R$ - factors based on ALL data will be even larger.

Fractional atomic coordinates and isotropic or equivalent isotropic displacement parameters $\left(\AA^{2}\right)$

\begin{tabular}{lllll}
\hline & $x$ & $y$ & $z$ & $U_{\text {iso }} * U_{\text {eq }}$ \\
\hline Fe1 & 0.0000 & 0.0000 & 0.0000 & $0.01099(17)$ \\
S1 & $0.63302(12)$ & $0.24660(11)$ & $0.21899(9)$ & $0.00929(18)$ \\
O1 & $0.3609(4)$ & $0.2280(4)$ & $0.0944(3)$ & $0.0142(4)$ \\
O2 & $0.8223(4)$ & $0.2674(3)$ & $0.1024(3)$ & $0.0131(4)$ \\
O3 & $0.6559(4)$ & $0.0411(3)$ & $0.3285(3)$ & $0.0130(4)$ \\
O4 & $0.6932(4)$ & $0.4606(3)$ & $0.3470(3)$ & $0.0122(4)$ \\
N1 & $0.0660(5)$ & $-0.1738(5)$ & $0.2599(4)$ & $0.0117(5)$ \\
H1A & $0.129(7)$ & $-0.079(7)$ & $0.350(5)$ & $0.017(9)^{*}$ \\
H1B & $-0.077(8)$ & $-0.249(7)$ & $0.265(5)$ & $0.022(10)^{*}$ \\
N2 & $0.2501(5)$ & $-0.3342(5)$ & $0.2854(4)$ & $0.0144(5)$ \\
H2A & $0.281(7)$ & $-0.403(7)$ & $0.390(6)$ & $0.021(10)^{*}$ \\
H2B & $0.391(8)$ & $-0.256(7)$ & $0.293(6)$ & $0.023(10)^{*}$ \\
H2C & $0.188(8)$ & $-0.442(7)$ & $0.204(6)$ & $0.026(11)^{*}$ \\
\end{tabular}

Atomic displacement parameters $\left(\AA^{2}\right)$

\begin{tabular}{lllllll}
\hline & $U^{11}$ & $U^{22}$ & $U^{33}$ & $U^{12}$ & $U^{13}$ & $U^{23}$ \\
\hline Fe1 & $0.0100(3)$ & $0.0120(3)$ & $0.0110(3)$ & $0.0012(2)$ & $0.0031(2)$ & $0.0007(2)$ \\
S1 & $0.0085(3)$ & $0.0102(3)$ & $0.0094(3)$ & $0.0007(2)$ & $0.0033(3)$ & $0.0001(3)$ \\
O1 & $0.0095(9)$ & $0.0156(10)$ & $0.0162(10)$ & $0.0006(8)$ & $0.0014(8)$ & $0.0011(8)$ \\
O2 & $0.0136(10)$ & $0.0131(10)$ & $0.0141(10)$ & $0.0020(8)$ & $0.0063(8)$ & $0.0013(8)$ \\
O3 & $0.0157(10)$ & $0.0118(10)$ & $0.0126(10)$ & $0.0017(8)$ & $0.0058(8)$ & $0.0024(8)$ \\
O4 & $0.0151(10)$ & $0.0112(10)$ & $0.0105(10)$ & $0.0009(8)$ & $0.0048(8)$ & $-0.0015(8)$ \\
N1 & $0.0079(11)$ & $0.0138(12)$ & $0.0136(12)$ & $0.0014(10)$ & $0.0033(10)$ & $0.0006(10)$ \\
N2 & $0.0151(13)$ & $0.0145(13)$ & $0.0133(13)$ & $0.0034(11)$ & $0.0023(11)$ & $0.0012(11)$ \\
\hline
\end{tabular}

Geometric parameters $\left(\AA,{ }^{\circ}\right)$

\begin{tabular}{llll}
\hline $\mathrm{Fe} 1-\mathrm{O} 1$ & $2.109(2)$ & $\mathrm{S} 1-\mathrm{O} 1$ & $1.513(2)$ \\
$\mathrm{Fe} 1-\mathrm{O} 1^{\mathrm{i}}$ & $2.109(2)$ & $\mathrm{O} 2-\mathrm{Fe}^{1 \mathrm{iv}}$ & $2.147(2)$ \\
$\mathrm{Fe} 1-\mathrm{O} 2^{\mathrm{ii}}$ & $2.147(2)$ & $\mathrm{N} 1-\mathrm{N} 2$ & $1.446(4)$ \\
$\mathrm{Fe} 1-\mathrm{O} 2^{\mathrm{iii}}$ & $2.147(2)$ & $\mathrm{N} 1-\mathrm{H} 1 \mathrm{~A}$ & $0.82(4)$ \\
$\mathrm{Fe} 1-\mathrm{N} 1$ & $\mathrm{~N} 1-\mathrm{H} 1 \mathrm{~B}$ & $0.82(4)$ \\
$\mathrm{Fe} 1-\mathrm{N} 1^{\mathrm{i}}$ & $2.184(2)$ & $\mathrm{N} 2-\mathrm{H} 2 \mathrm{~A}$ & $0.88(4)$ \\
$\mathrm{S} 1-\mathrm{O} 2$ & $1.184(2)$ & $\mathrm{N} 2-\mathrm{H} 2 \mathrm{~B}$ & $0.80(4)$ \\
$\mathrm{S} 1-\mathrm{O} 3$ & $1.464(2)$ & $\mathrm{N} 2-\mathrm{H} 2 \mathrm{C}$ & $0.82(4)$ \\
$\mathrm{S} 1-\mathrm{O} 4$ & $1.473(2)$ & & \\
& $1.482(2)$ & $\mathrm{O} 2-\mathrm{S} 1-\mathrm{O} 1$ & $108.74(12)$ \\
$\mathrm{O} 1-\mathrm{Fe} 1-\mathrm{O} 1^{\mathrm{i}}$ & 180.0 & $\mathrm{O} 3-\mathrm{S} 1-\mathrm{O} 1$ & $111.25(12)$ \\
$\mathrm{O} 1-\mathrm{Fe} 1-\mathrm{O} 2^{\mathrm{ii}}$ & $87.04(8)$ & &
\end{tabular}




\begin{tabular}{|c|c|c|c|}
\hline $\mathrm{O} 1^{\mathrm{i}}-\mathrm{Fe} 1-\mathrm{O} 2^{\mathrm{ii}}$ & $92.96(8)$ & $\mathrm{O} 4-\mathrm{S} 1-\mathrm{O} 1$ & $109.43(12)$ \\
\hline $\mathrm{O} 1-\mathrm{Fe} 1-\mathrm{O} 2^{\mathrm{iii}}$ & $92.96(8)$ & $\mathrm{S} 1-\mathrm{O} 1-\mathrm{Fe} 1$ & $142.94(13)$ \\
\hline 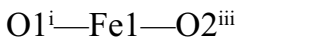 & $87.04(8)$ & $\mathrm{S} 1-\mathrm{O} 2-\mathrm{Fe}^{\mathrm{iv}}$ & $128.85(12)$ \\
\hline $\mathrm{O} 2^{\mathrm{ii}}-\mathrm{Fe} 1-\mathrm{O} 2^{\mathrm{iii}}$ & 180.0 & $\mathrm{~N} 2-\mathrm{N} 1-\mathrm{Fe} 1$ & $118.02(18)$ \\
\hline $\mathrm{O} 1-\mathrm{Fe} 1-\mathrm{N} 1$ & $90.79(9)$ & $\mathrm{N} 2-\mathrm{N} 1-\mathrm{H} 1 \mathrm{~A}$ & $102(3)$ \\
\hline $\mathrm{O} 1 \mathrm{i}-\mathrm{Fe} 1-\mathrm{N} 1$ & $89.21(9)$ & $\mathrm{Fe} 1-\mathrm{N} 1-\mathrm{H} 1 \mathrm{~A}$ & $111(3)$ \\
\hline $\mathrm{O} 22^{\mathrm{ii}}-\mathrm{Fe} 1-\mathrm{N} 1$ & $95.14(9)$ & $\mathrm{N} 2-\mathrm{N} 1-\mathrm{H} 1 \mathrm{~B}$ & $107(3)$ \\
\hline 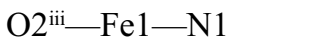 & $84.86(9)$ & $\mathrm{Fe} 1-\mathrm{N} 1-\mathrm{H} 1 \mathrm{~B}$ & $107(3)$ \\
\hline $\mathrm{O} 1-\mathrm{Fe} 1-\mathrm{N} 1^{\mathrm{i}}$ & $89.21(9)$ & $\mathrm{H} 1 \mathrm{~A}-\mathrm{N} 1-\mathrm{H} 1 \mathrm{~B}$ & $112(4)$ \\
\hline 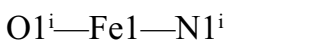 & $90.79(9)$ & $\mathrm{N} 1-\mathrm{N} 2-\mathrm{H} 2 \mathrm{~A}$ & $119(3)$ \\
\hline 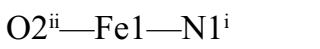 & $84.86(9)$ & $\mathrm{N} 1-\mathrm{N} 2-\mathrm{H} 2 \mathrm{~B}$ & $106(3)$ \\
\hline $\mathrm{O} 2^{\mathrm{iii}}-\mathrm{Fe} 1-\mathrm{N} 1^{\mathrm{i}}$ & $95.14(9)$ & $\mathrm{H} 2 \mathrm{~A}-\mathrm{N} 2-\mathrm{H} 2 \mathrm{~B}$ & $101(4)$ \\
\hline $\mathrm{N} 1-\mathrm{Fe} 1-\mathrm{N} 1^{\mathrm{i}}$ & 180.0 & $\mathrm{~N} 1-\mathrm{N} 2-\mathrm{H} 2 \mathrm{C}$ & $106(3)$ \\
\hline $\mathrm{O} 2-\mathrm{S} 1-\mathrm{O} 3$ & $109.79(12)$ & $\mathrm{H} 2 \mathrm{~A}-\mathrm{N} 2-\mathrm{H} 2 \mathrm{C}$ & $105(4)$ \\
\hline $\mathrm{O} 2-\mathrm{S} 1-\mathrm{O} 4$ & $107.99(12)$ & $\mathrm{H} 2 \mathrm{~B}-\mathrm{N} 2-\mathrm{H} 2 \mathrm{C}$ & $122(4)$ \\
\hline $\mathrm{O} 3-\mathrm{S} 1-\mathrm{O} 4$ & $109.57(12)$ & & \\
\hline $\mathrm{O} 2-\mathrm{S} 1-\mathrm{O} 1-\mathrm{Fe} 1$ & $114.0(2)$ & $\mathrm{O} 3-\mathrm{S} 1-\mathrm{O} 2-\mathrm{Fe}^{\mathrm{iv}}$ & $24.58(19)$ \\
\hline $\mathrm{O} 3-\mathrm{S} 1-\mathrm{O} 1-\mathrm{Fe} 1$ & $-7.1(3)$ & $\mathrm{O} 4-\mathrm{S} 1-\mathrm{O} 2-\mathrm{Fe}^{1 \mathrm{iv}}$ & $143.99(14)$ \\
\hline $\mathrm{O} 4-\mathrm{S} 1-\mathrm{O} 1-\mathrm{Fe} 1$ & $-128.3(2)$ & $\mathrm{O} 1-\mathrm{S} 1-\mathrm{O} 2-\mathrm{Fe} 1^{\mathrm{iv}}$ & $-97.35(16)$ \\
\hline 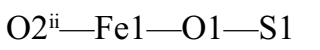 & $124.1(2)$ & $\mathrm{O} 1-\mathrm{Fe} 1-\mathrm{N} 1-\mathrm{N} 2$ & $-76.0(2)$ \\
\hline 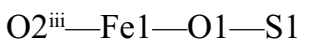 & $-55.9(2)$ & $\mathrm{O} 1-\mathrm{Fe} 1-\mathrm{N} 1-\mathrm{N} 2$ & $104.0(2)$ \\
\hline $\mathrm{N} 1-\mathrm{Fe} 1-\mathrm{O} 1-\mathrm{S} 1$ & $29.0(2)$ & 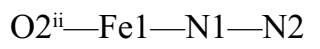 & $-163.1(2)$ \\
\hline $\mathrm{N} 1{ }^{\mathrm{i}}-\mathrm{Fe} 1-\mathrm{O} 1-\mathrm{S} 1$ & $-151.0(2)$ & 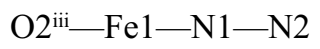 & $16.9(2)$ \\
\hline
\end{tabular}

Symmetry codes: (i) $-x,-y,-z$; (ii) $x-1, y, z$; (iii) $-x+1,-y,-z$; (iv) $x+1, y, z$.

Hydrogen-bond geometry $\left(A,{ }^{\circ}\right)$

\begin{tabular}{lllll}
\hline$D-\mathrm{H} \cdots A$ & $D-\mathrm{H}$ & $\mathrm{H} \cdots A$ & $D \cdots A$ & $D-\mathrm{H}^{\cdots} A$ \\
\hline $\mathrm{N} 1-\mathrm{H} 1 A^{\cdots} \cdots 3^{\mathrm{v}}$ & $0.82(4)$ & $2.37(4)$ & $3.070(3)$ & $143(3)$ \\
$\mathrm{N} 1-\mathrm{H} 1 B \cdots{ }^{\mathrm{vi}}$ & $0.82(4)$ & $2.12(4)$ & $2.867(3)$ & $151(4)$ \\
$\mathrm{N} 2-\mathrm{H} 2 A \cdots 4^{\mathrm{v}}$ & $0.88(4)$ & $1.96(4)$ & $2.799(3)$ & $160(4)$ \\
$\mathrm{N} 2-\mathrm{H} 2 B \cdots \mathrm{O} 3$ & $0.80(4)$ & $2.02(4)$ & $2.769(4)$ & $156(4)$ \\
$\mathrm{N} 2-\mathrm{H} 2 C \cdots \mathrm{O} 2^{i i i}$ & $0.82(4)$ & $2.51(4)$ & $2.849(3)$ & $106(3)$ \\
$\mathrm{N} 2-\mathrm{H} 2 C \cdots \mathrm{O} 2^{\mathrm{vi}}$ & $0.82(4)$ & $2.32(4)$ & $3.011(4)$ & $141(4)$ \\
$\mathrm{N} 2-\mathrm{H} 2 C \cdots \mathrm{O} 1^{\mathrm{vii}}$ & $0.82(4)$ & $2.45(4)$ & $3.073(3)$ & $133(4)$
\end{tabular}

Symmetry codes: (iii) $-x+1,-y,-z$; (v) $-x+1,-y,-z+1$; (vi) $x-1, y-1, z$; (vii) $x, y-1, z$. 